\title{
Aurore GIACOMEL, Les enjeux du travail émotionnel individuel et collectif dans les groupes hôteliers multinationaux : la complexité de l'équilibre émotionnel au service de l'homéostasie organisationnelle
}

Thèse de doctorat en sciences de gestion de l'Université d'Angers, dirigée par Dominique Peyrat-Guillard (soutenue le 18 septembre 2019)

The issues of emotional labor in hospitality multinational groups: the individual and collective emotional balance at the service of organizational homeostasis

\section{Aurore Giacomel}

\section{OpenEdition}

\section{Journals}

Édition électronique

URL : http://journals.openedition.org/tourisme/2471

DOI : $10.4000 /$ tourisme. 2471

ISSN : 2492-7503

Éditeur

Éditions touristiques européennes

Référence électronique

Aurore Giacomel, «Aurore giacomel, Les enjeux du travail émotionnel individuel et collectif dans les groupes hôteliers multinationaux : la complexité de l'équilibre émotionnel au service de l'homéostasie organisationnelle », Mondes du Tourisme [En ligne], 16 | 2019, mis en ligne le 01 décembre 2019, consulté le 24 septembre 2020. URL : http://journals.openedition.org/tourisme/2471 ; DOI : https:// doi.org/10.4000/tourisme.2471

Ce document a été généré automatiquement le 24 septembre 2020.

\section{c)}

Mondes du tourisme est mis à disposition selon les termes de la licence Creative Commons Attribution - Pas d'Utilisation Commerciale - Pas de Modification 4.0 International. 


\section{Aurore GIACOMEL, Les enjeux du travail émotionnel individuel et collectif dans les groupes hôteliers multinationaux : la complexité de l'équilibre émotionnel au service de l'homéostasie organisationnelle}

Thèse de doctorat en sciences de gestion de l'Université d'Angers, dirigée par Dominique Peyrat-Guillard (soutenue le 18 septembre 2019)

The issues of emotional labor in hospitality multinational groups: the individual and collective emotional balance at the service of organizational homeostasis

\section{Aurore Giacomel}

Notre époque se caractérise par un renouvellement éthique et écologique, plus respectueux de l'humain et de l'environnement. L'expérience du touriste est au cœur des préoccupations, notamment au sein des groupes hôteliers multinationaux. Progressivement, l'accent mis sur l'expérience vécue pour attirer et retenir les clients se prolonge de l'autre côté du desk, avec le développement d'une «expérience collaborateur » de plus en plus poussée. En effet, les salariés du front office sont incités à « rester eux-mêmes » dans leur travail, afin d'afficher une image plus authentique face aux clients. Cette injonction s'ajoute à celle de l'hyperpersonnalisation du service : le salarié doit paradoxalement correspondre à ce que chaque client attend, tout en restant lui-même. De telles injonctions contradictoires génèrent une tension constante, qui peut engendrer des comportements de retrait et des déséquilibres émotionnels chez les salariés.

Dans ce contexte de travail, pourquoi certains salariés subissent ces injonctions, jusqu'à ressentir de la souffrance au travail, alors que d'autres s'en accommodent 
parfaitement? Sur quels facteurs l'organisation peut-elle jouer pour garantir simultanément le bien-être de ses salariés et la qualité du service ? Notre hypothèse est que la cohérence des attentes, perçues et réelles, des clients et des employés a un impact sur les effets des pratiques $\mathrm{RH}$, dont la compréhension est atteignable principalement via les mécanismes psychosociaux des individus. Les discours des firmes hôtelières tournent aujourd'hui autour de la flexibilité, l'agilité et l'hyperpersonnalisation, en faveur d'une adaptabilité accrue. Cependant, ces principes sont mis en œuvre de façon peu réaliste, ce qui crée des dysfonctionnements à tous les niveaux de l'organisation. Ainsi, les firmes semblent créer elles-mêmes les phénomènes qu'elles essaient de contrer : un désengagement des clients, qui ne retrouvent pas la promesse dans la prestation effective, et un désengagement des salariés, confrontés aux demandes paradoxales de leur rôle de travail. Nous savons que les pratiques RH d'amélioration du bien-être influent positivement sur la rétention et l'implication des salariés; cependant, les mécanismes qui constituent ces liens sont peu étudiés. Il semble notamment primordial d'examiner les mécanismes émotionnels qui entrent en jeu, particulièrement dans le cadre de métiers qui gèrent de l'humain.

3 La littérature sur le travail émotionnel (TE) englobe ces aspects propres aux métiers dits du front office. Hochschild (1983) a initialement défini le concept comme «la manière de gérer ses émotions pour se donner une apparence physique correspondant à ce qui est attendu socialement ; celui-ci a lieu en échange d'un salaire » (Hochschild, 1983, p. 7). Afin d'afficher l'émotion adéquate, l'employé peut utiliser trois stratégies de TE : exprimer naturellement ses émotions, réaliser un jeu en surface (feinte émotionnelle) ou réaliser un jeu en profondeur (transformation de l'émotion). Depuis, de nombreux auteurs se sont attelés à l'étude de ce concept, cherchant à discerner des liens entre les stratégies émotionnelles utilisées par les salariés et leurs comportements et attitudes au travail. La littérature souffre néanmoins d'un morcellement de l'étude du concept, qui n'a pas permis la formation d'un consensus sur sa définition et empêche d'appréhender la gestion émotionnelle des salariés de façon réaliste. En outre, les échelles mesurent, chacune, des composantes différentes du TE, et donc un TE de nature différente. Ainsi, les chercheurs évaluent indistinctement un processus, un mécanisme biologique, une compétence, une expression ou encore une stratégie, des mesures qui n'impliquent pas les mêmes outils et ne mènent pas aux mêmes conclusions.

Ce travail de thèse propose de relier la stratégie hôtelière aux métiers du front office, grâce à un cadre épistémologique original en sciences de gestion : la pensée complexe d'Edgar Morin. Adopter le paradigme de la complexité pour étudier le TE a permis d'explorer ses divers aspects disciplinaires (neurobiologiques, psychologiques, sociologiques, etc.) et de prendre en compte simultanément les différents niveaux d'analyse (individuel, interindividuel, organisationnel). L'objectif est d'identifier le potentiel que peuvent offrir les capacités complexes humaines d'autorégulation en vue du maintien durable de l'organisation dans un environnement complexe. Notre étude compile plusieurs objets de recherche complexes et imbriqués: les mécanismes émotionnels chez l'être humain, en situation de travail, au sein d'une organisation touristique, dans une société en mutation. La problématique posée est la suivante : une meilleure compréhension du TE et de l'équilibre émotionnel individuel et collectif au sein des équipes en contact avec les clients peut-elle permettre d'optimiser la capacité adaptative dans les métiers du front office de l'hôtellerie? À partir d'une littérature transdisciplinaire sur la régulation et la gestion des émotions, nous avons fait émerger 
un modèle représentant la complexité du TE ( $c f$. figure 1 ). Ce modèle s'appuie sur de multiples théories en biologie et neurosciences, en sociologie, en psychologie et psychanalyse, en comportements organisationnels et en systémique. Nous avons réalisé 60 entretiens semi-directifs et des observations dans des équipes de réception et de restauration en salle, au sein de cinq Novotel en région parisienne. Après analyse textuelle, à l'aide du logiciel IraMuTeQ, de nouveaux éclairages apparaissent quant aux comportements et vécus émotionnels dans ces métiers.

Figure 1. Le travail émotionnel comme système complexe

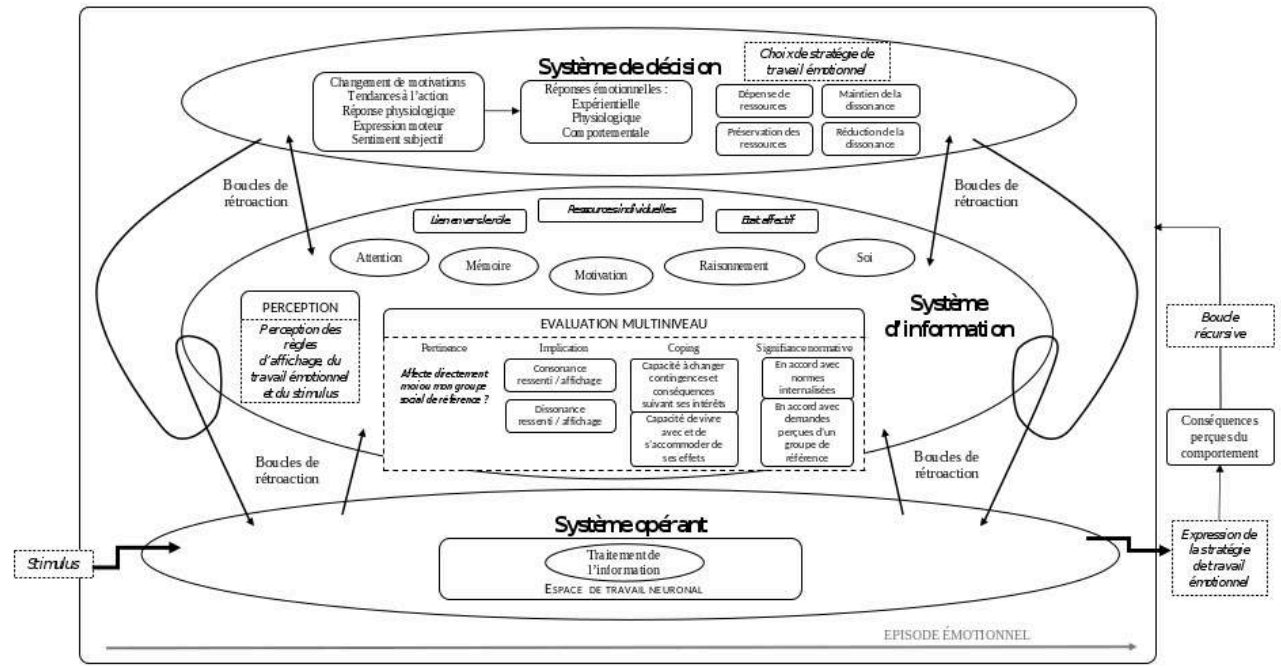

Contrairement à notre hypothèse de départ, selon laquelle les nouvelles normes émotionnelles imposées aux salariés par les hôteliers participeraient à l'augmentation des troubles émotionnels parmi le personnel de front office, nos analyses désignent plutôt comme coupables des normes d'organisation du travail, des normes liées à la digitalisation et des normes de communication interne et externe. En effet, le maintien volontaire d'un fort turnover et des situations fréquentes de sous-effectif dans les équipes de contact réduisent l'espace temporel destiné à évacuer les émotions et le soutien dans l'équipe et nécessitent donc un effort de TE supplémentaire. La vocation des outils numériques à réduire la place des procédures administratives au profit d'une relation client approfondie entraîne, au contraire, une réduction des contacts clients et prive les salariés de reconnaissance directe par les clients. Ajoutons à cela une crainte généralisée des réceptionnistes de voir leur emploi remplacé à l'avenir par les technologies. Côté ressources humaines, la focalisation du recrutement sur le savoirêtre est jugée chronophage par les équipes, qui doivent se charger de la formation des nouvelles recrues aux savoir-faire. Parallèlement, les pratiques de management participatif ne prennent réellement en compte ni les besoins ni les contraintes des employés sur le terrain. Ceci engendre des ressentis de déception et de trahison, que doivent gérer les salariés. Pour finir, les promesses d'hyperpersonnalisation du service accroissent les exigences des clients et demandent donc un effort de TE supplémentaire. De même, l'allègement des contraintes sociales pour les clients, incités à se comporter à l'hôtel comme chez eux, favorise la généralisation d'un manque de respect des clients envers les salariés, à gérer en supplément. 
Plusieurs résultats importants sur la nature et le fonctionnement du TE sont apparus. Tout d'abord, un TE peut être réalisé lors de tout épisode émotionnel, qu'il soit lié à une interaction interpersonnelle ou non. Chez les répondants, même ceux ayant un recul manifeste sur leur gestion émotionnelle, l'identification des stratégies de jeu en surface et de jeu en profondeur, usuelles dans la littérature scientifique, ne nous a pas paru évidente. Deux processus de gestion émotionnelle sont principalement vécus sur le terrain: le fait de bloquer directement le négatif chez le client, par habitude ou en relativisant, car le salarié sait qu'il va résoudre le problème du client ; mettre le négatif en attente pour se concentrer sur le présent et évacuer les émotions ensuite. Sur sept profils de combinaison des stratégies de TE résultant de nos analyses, trois profils ne font pas référence aux stratégies décrites par la littérature. Enfin, ces différentes façons de réaliser un TE se répercutent sous forme de vécus et de comportements au travail, que nous avons pu catégoriser en neuf profils. En l'état, aucun lien significatif n'a été trouvé entre ces deux types de profil. Nos résultats font également émerger l'existence d'un TE collectif. En effet, en front office, l'accueil d'un client se fait par le collectif " équipe de réception » et non par un réceptionniste; et l'équipe gère ses émotions collectivement comme s'il s'agissait d'un seul organisme.

7 L'impact qu'auront les normes organisationnelles sur les comportements et vécus au travail dépendra directement de leur influence sur le TE réalisé par les salariés, luimême déterminé par le lien que l'individu entretient envers son rôle de travail, ses ressources individuelles ainsi que les ressources matérielles disponibles sur le lieu de travail. Nous avons décelé un quatrième antécédent du TE : le lien collectif de l'équipe de front office envers leur rôle collectif de travail. Le lien individuel envers le rôle de travail dépendra du degré d'internalisation des normes organisationnelles: plus l'individu aura intégré ces normes, plus il s'identifiera à son rôle de travail. Le type de lien développé serait fonction de : la marge de manœuvre par rapport au rôle prescrit, la cible du lien, les apports du rôle par rapport à soi et les raisons de l'attachement au rôle. Les ressources individuelles, consciemment mobilisées par les répondants, sont: les capacités de contrôle sur ses émotions - facilitées par l'existence d'espaces prévus pour la gestion émotionnelle et par certains traits de personnalité - ; la maîtrise perçue de son travail et de ses émotions ; le plaisir et les relations interpersonnelles au travail ; les compétences relationnelles; le ressourcement hors travail; l'équilibre entre vie privée et vie professionnelle. Ainsi, les ressources matérielles du lieu de travail, les ressources individuelles, le lien individuel envers le rôle de travail et le lien collectif envers le rôle collectif de travail paraissent constituer quatre piliers de l'équilibre émotionnel au travail.

8 L'être humain est une entité adaptative, aussi bien au niveau moléculaire, cellulaire et neuronal qu'au niveau psychologique et même social. Or, les organisations, qui cherchent à contrôler le moindre rouage de la formidable machine qu'est l'humain notamment le système des émotions - comme s'il s'agissait d'une machine artificielle, ne font qu'empêcher les capacités adaptatives du vivant. En effet, contrairement à la machine vivante qui s'autorégénère continûment, poussée vers un équilibre homéostatique qu'elle se doit de conserver afin de survivre dans le temps, la machine artificielle ne peut croître que si l'on y ajoute des rouages supplémentaires et dysfonctionne dès qu'une de ses pièces dysfonctionne. Toute organisation complexe peut, sur le modèle de la machine vivante, s'adapter de façon autonome en fonction des stimuli reçus de l'extérieur et ainsi s'adapter à son environnement en tirant parti de ses 
capacités de réorganisation interne. L'homéostasie organisationnelle constituerait, en ce sens, un objectif alternatif à l'objectif d'expansion infinie des firmes, qui arrive aux limites de ses possibles aujourd'hui. Il s'agirait, pour les entreprises, non plus de croître indéfiniment, mais de tendre vers un équilibre qui puisse être durable et utile pour l'humain, en tant que société et individu.

\section{BIBLIOGRAPHIE}

Arlie HOCHSCHILD, The Managed Heart: Commercialization of Human Feeling, University of California Press, 1983.

\section{RÉSUMÉS}

La complexité croissante de l'environnement exige une capacité accrue d'adaptation des chaînes hôtelières, qui fondent leurs stratégies sur les demandes d'expériences et d'authenticité des touristes. Les salariés du front office sont, en effet, incités à répondre à des attentes paradoxales face aux clients, ce qui influe sur leur travail émotionnel et peut déboucher sur des comportements de retrait et des cas de déséquilibre émotionnel. Une meilleure compréhension du travail émotionnel et de l'équilibre émotionnel individuel et collectif peut-elle permettre d'optimiser la capacité adaptative dans ces métiers de contact? Nous proposons une analyse qualitative et transdisciplinaire, guidée par l'approche épistémologique de la pensée complexe d'Edgar Morin, afin de traiter ce questionnement complexe.

\section{AUTEUR}

\section{AURORE GIACOMEL}

ATER, Université d'Angers

Docteure en sciences de gestion

aurore.giacomel@univ-angers.fr 